Geopolítica(s) Revista de estudios sobre espacio y poder ISSN: 2172-3958

\title{
Planificación, políticas territoriales y competencias de los gobernadores de los estados en la reciente dinámica del federalismo brasileño: el caso de Bahia
}

\author{
Vanessa da Silva Vieira ${ }^{1}$ y Rubén Camilo Lois González ${ }^{2}$
}

Recibido: 7 de diciembre de 2016 / Aceptado: 22 de agosto de 2018

Resumen. En este trabajo, se considera importante debatir sobre la temática del federalismo abordado desde el punto de vista de la Geografía Política y la Geopolítica. Así, la intención principal es analizar el proceso de territorialización dirigido por el gobierno de cada unidad federativa (los estados brasileños), con el objetivo de legitimar su capacidad de acción autónoma dentro de la federación. Desde esta perspectiva, la cuestión central es: ¿de qué forma el gobierno de las unidades federativas se comportan ante la autonomía que les fue concedida, para elaborar y ejecutar estrategias de inversiones en su espacio de gestión? El objeto de estudio será el estado de Bahía, que se analizará considerando dos períodos gubernamentales, 2003-2006 y 2007-2010 respectivamente, cuando se elaboraron y ejecutaron propuestas bien diferenciadas para el desarrollo del territorio.

Palabras clave: Brasil; federalismo; territorialización; políticas públicas; divisiones territoriales.

\section{[en] Planning, Territorial Policies and Areas of Competence of States' Governors in the Recent Federalist Dynamic in Brazil: The Case of Bahia}

\begin{abstract}
This work considers that it is important to debate the question of federalism from the point of view of Political Geography and Geopolitics. The article's main goal is to analyse the territorialization processes conducted by the governments of each federal unit (the Brazilian states) in order to legitimize their capacity of autonomous action within the federation. From this standpoint, the key question turns out to be 'How do the governments of the federal units handle their autonomy to elaborate and execute investment strategies within their territories?' The paper focuses on the state of Bahia, which will be analysed in relation to two terms, 2003-2006 and 2007-2010, during which two clearly distinct territorial development plans were elaborated and put in place, respectively.
\end{abstract}

Keywords: Brazil; federalism; territorialization; public policy; territorial divisions.

[pt] Planejamento, políticas territoriais e competências dos governadores dos estados na recente dinâmica do federalismo brasileiro: o caso de Bahia

1 Departamento de Geografia, Universidade Estadual de Feira de Santana (Brasil)

E-mail: vsvuefs@gmail.com

2 Departamento de Xeografía, Facultade de Xeografía e Historia, Universidade de Santiago de Compostela.

E-mail: rubencamilo.lois@usc.es 
Resumo. Neste artigo, considera-se importante discutir o tema do federalismo abordado do ponto de vista da Geografia Política e Geopolítica. Assim, a intenção principal é analisar o processo de territorialização dirigido pelo governo de cada unidade federativa (os estados brasileiros), com o objetivo de legitimar sua capacidade de ação autônoma dentro da federação. Nessa perspectiva, a questão central é: como o governo das unidades federativas se comporta diante da autonomia que lhes foi concedida, para elaborar e executar estratégias de investimento em seu espaço de gestão? O objeto de estudo será o estado da Bahia, que será analisado considerando dois períodos de governo, 2003-2006 e 20072010, respectivamente, quando propostas bem diferenciadas para o desenvolvimento do território foram elaboradas e executadas

Palavras-chave: Brasil, federalismo, territorialização, políticas públicas, divisões territoriais.

Sumario. Introducción. 1. Federalismo en la dimensión espacial: agenda actual y propuestas para la ampliación del debate en Geografía. 2. Federalismo y las especificidades en el contexto brasileño. 3. Políticas territoriales en el contexto federativo: El Plan Plurianual (PPA). 3.1. Los Planes Plurianuales en Bahía. 3.2. El PPA 2004-2007. 3.3. El PPA 2008-2011. Consideraciones finales. Bibliografía.

Cómo citar: Vieira, Vanessa da Silva, y Lois González, Rubén Camilo (2018) “Planificación, políticas territoriales y competencias de los gobernadores de los estados en la reciente dinámica del federalismo brasileño: el caso de Bahía”. Geopolítica(s). Revista de estudios sobre espacio y poder, vol. 9, núm. 2, 209-230.

\section{Introducción}

En Brasil, los análisis sobre el sistema de gobierno federativo concentran esfuerzos en los aspectos económico, tributario y fiscal y/o en la conflictiva relación del poder entre los entes de la federación, descuidando a menudo la espacialidad y las implicaciones geopolíticas de esta temática. Por eso, ahora se procura ampliar el debate generado mediante un acercamiento desde la Geografía Política que concibe al federalismo como una organización social, política y espacial, que influye directamente en la dinámica del territorio. Además, la Geografía Política subraya los mecanismos institucionales de regulación, al incluir en el análisis, las leyes, decretos, programas y planes como instrumentos que regulan la acción gubernamental sobre el territorio (Fonseca, 2005). De esta forma, se añade en este debate el Plan Plurianual (PPA) que, desde la Constitución Federal Brasileña de 1988, es el primer instrumento de planificación de gestión pública, y establece constitucionalmente prioridades para el uso de los recursos financieros. Además, la ejecución del PPA depende de una base espacial, traducida en una regionalización específica por la que cada gobierno expresa sus prioridades para las políticas territoriales. Por supuesto, aplica una determinada lectura intencional en el territorio buscando tanto su reequilibrio interno como organizarlo según sus principios de gestión (Boisier, 1985; Agnew, 1987; Raffestin, 1993).

Sea abordado con un enfoque más competitivo o más participativo, el hecho es que los gobiernos estaduales utilizan cada vez más los PPAs, con el fin de orientar mejor las competencias que se les atribuyen dentro del pacto federativo. Desde el punto de vista espacial, se destacan las siguientes competencias: a) establecer mecanismos para atraer inversiones mediante la división de zonas y la formación de territorios; b) delimitar la forma en la que participa la población en la planificación de las políticas territoriales; c) regionalizar el territorio para la ejecución de los programas y distribución de presupuesto. De este modo, la cuestión central de este 
artículo es: ¿de qué forma el gobierno de las unidades federativas se comporta ante la autonomía que se les concedió para elaborar y ejecutar estrategias de inversiones en su espacio de gestión?

Este enunciado es la auténtica pregunta de investigación, a partir de la cual se organiza nuestro estudio. De hecho, los gobiernos estaduales del período democrático han diseñado un auténtico mapa de sus respectivos territorios a partir de la escala supralocal. La opción adoptada para estas territorializaciones (áreas metropolitanas, territorios atractivos para captar inversiones, territorios de identidad, etc.), hasta dónde expresa la lectura ideológica del gobierno a nivel espacial. Asimismo, ¿de qué forma repercute en la integración de los respectivos estados en el espacio económico brasileño, que se define como emergente o en crecimiento? En este contexto, ¿qué especificidades muestran estas políticas en los estados nordestinos, tradicionalmente caracterizados como pobres y poco desarrollados? Y, finalmente, ¿ constituye el estado de Bahía un buen ejemplo de las prácticas materializadas en el Nordeste brasileño?

El objeto de investigación es Bahía, estado brasileño que históricamente se caracterizó por una organización espacial definida por desequilibrios regionales y, que también acusó una tenue integración urbano-regional. El objetivo es comprender, a partir de una perspectiva comparativa, las prioridades que contiene el plan de cada gobierno al delimitar los espacios para su acción. Por lo tanto, se utiliza como instrumento de análisis el Plan Plurianual de Bahía, específicamente, el PPA 20042007 y el PPA 2008-2011, del Gobierno de Paulo Souto (2003-2006, de la derecha tradicional) y del Gobierno de Jaques Wagner (2006-2010, del PT) respectivamente. El estudio del contenido de los PPAs se centra sobre todo en su articulado e implicaciones territoriales, sin entrar a considerar las lógicas planificadoras que los sucesivos gobiernos federales aplicaban sobre los distintos estados en esos años o sin valorar aquellas políticas que municipios de cierto tamaño ejecutaron en sus demarcaciones. El análisis de la complementariedad/competencia de los diferentes niveles de gobierno territorial en el Brasil del primer decenio del siglo XXI desborda la finalidad de este trabajo y obligará a realizar análisis detallados posteriores.

Este artículo se divide en tres partes, además de la introducción y las consideraciones finales. La primera se refiere al trato que la Geografía ha dado al tema del federalismo, la segunda abarca el federalismo de una forma más amplia, su origen, características, la forma en la que se presenta en el contexto brasileño y su influencia en la acción de los gobernadores. Por último, la tercera aborda las unidades de planificación definidas por los gobiernos de Paulo Souto y Jaques Wagner, las cuales se institucionalizaron a través de los respectivos Planes Plurianuales y que revelan la forma de apropiación del espacio, delimitando territorios para, por ejemplo, distribuir recursos e inversiones.

\section{Federalismo en la dimensión espacial: agenda actual y propuestas para la ampliación del debate en Geografía}

En Brasil, el federalismo ha sido estudiado frecuentemente, sobre todo en el campo de las ciencias políticas (Abrucio, 2005, 1999, 1994; Arretche, 2010, 2009; Souza, 
2013, 2008, 2005, 2003) y la economía (Affonso, 2000; Rezende, 2009, 2007, 2006, 1995). De hecho, los estudios han revelado varios matices del sistema federativo brasileño desde su implantación en 1891. Sin embargo, todavía queda un ámbito provechoso para el análisis de la dimensión espacial del federalismo y, la Geografía es una disciplina adecuada para tal efecto (Claval, 1978; Paasi, 2002).

Existen, al menos, dos motivaciones geográficas. La primera consiste en un “acuerdo de base territorial”, como afirma Castro (2005: 164), una “ingeniería política” que surge para solucionar las divergencias del poder político en territorios específicos y, permite "[...] acomodar conflictos y establecer alianzas sin que el Estado pierda su prerrogativa de centralidad política para todo el territorio" (Castro, 2005: 130). No obstante, a pesar de los acuerdos, el conflicto de competencias y el riesgo de supremacía de un poder sobre los demás son evidentes y exigen constantes actos de cooperación entre las diferentes escalas del gobierno. La segunda es una organización social política que se proyecta en diversas escalas. En base a Castro (1997: 36) “[...] la escala de observación define el fenómeno, lo que es visible en él y las posibilidades para su medida, análisis y observación [...] lo real está reflejado en cada una de esas escalas”. Si la escala da visibilidad al fenómeno, es fundamental destacar de qué forma el federalismo determina la distancia espacial en cada una de las escalas federativas. Sin duda, como apuntaba P. Taylor, las niveles local y supralocal son fundamentales en la lectura geopolítica del territorio, que juega con conceptos como rural-urbano, identidad e identidades, desarrollo desigual y juego político de los actores públicos (Taylor y Flint, 2002). También coincidiendo con la importancia de la escala, R. Haersbaert (2007, 2011), insiste en la vigencia de una organización territorial a diferentes escalas, que desmentiría la idea de que la globalización tiende a uniformizar el espacio, En este sentido, se refuerza la noción geográfica y política de la relevancia de las escalas supralocales y, para analizarlas, la interpretación teórica se ha construido a partir de diferentes lecturas realizadas por la geografía brasileña y latinoamericana recientemente (García Delgado, 1997; Díez, Gutiérrez y Pazzi, 2013).

Para acercarnos a los temas de investigación planteados, se ha realizado un planteamiento metodológico claro. Por una parte, se ha acometido una intensa revisión bibliográfica sobre el federalismo brasileño y, en especial, la cuestión nordestina, un conjunto de estados que se califican como menos desarrollados (con Bahía como ejemplo relevante), necesitados de acciones específicas destinadas a su modernización y crecimiento. Por otra, se ha trabajado en profundidad sobre los documentos oficiales emitidos por el gobierno de Bahía en los diferentes períodos considerados. Se analiza su contenido y la interpretación subyacente del desarrollo territorial que realizan. Junto a estos documentos, se ha utilizado la cartografía publicada como base para la lectura territorial. Por último, a partir de los discursos de los gobernadores y de los documentos que han producido se aplican métodos cualitativos de interpretación de los discursos ideológico-territoriales de fondo que se derivan de ellos. En definitiva, se trabaja con material generado por la acción de gobierno, para mediante un estudio crítico en profundidad entender sus formas de organización espacial dominantes.

En este artículo, nos interesamos especialmente por la escala estatal y el papel de los gobernadores, por dos motivos: 1) la importancia que la mencionada escala posee en la interlocución entre el municipio y la unión; 2) la importancia de los 
gobernadores estatales en la organización de los municipios por unidades regionales, para ejecutar las políticas territoriales. Se ha encontrado, en el campo de la geografía brasileña, una única publicación que incluye a los estados en el debate. Se trata de la correspondiente a Egler y Mattos (2003), cuyo eje principal de discusión son las posibilidades generadas por el federalismo para el desarrollo regional, a partir de las Regiones Integradas de Desarrollo (RIDE); un tema que ofrece la oportunidad de contemplar acciones de cooperación entre los estados pues, a pesar de no tratarse de un modelo de cooperación exclusivamente interestatal, depende del consentimiento de los estados involucrados. Con el objetivo de contribuir a los estudios en la escala estatal y ampliar la comprensión sobre las competencias de los gobernadores, la última sección de este artículo versa sobre las estrategias que los gobiernos del estado de Bahía (Paulo Souto, en el período de 2003-2006 y Jaques Wagner, en 2007-2010) materializaron, teniendo en cuenta su autonomía como ente federado.

\section{Federalismo y las especificidades en el contexto brasileño}

En 1748, Montesquieu publicó la obra L'esprit des Lois y, en ella concibe una República como un modelo de constitución de gobierno que "[...] posee todas las ventajas internas del gobierno republicano y la fuerza externa de la monarquía”. Para Montesquieu:

Esta forma de gobierno es una convención según la cual varios Cuerpos políticos consienten en convertirse en ciudadanos de un Estado mayor que pretenden formar. Es una sociedad de sociedades, que forman una nueva sociedad, que puede crecer con nuevos asociados que se unen a ella. (Montesquieu, 2005 [1748]: 62).

En este caso, Montesquieu se refiere al modelo de sistema federativo, con unidades independientes, que concuerdan con los procesos de centralización del poder político para el fortalecimiento del Estado. Ese fue el principio del modelo de federalismo adoptado en Estados Unidos y en algunos países europeos.

De forma muy directa, Elazar explica la "formula" del federalismo:

Los principios federales tienen que ver con la combinación de autogobierno y gobierno compartido. En el sentido más amplio, el federalismo implica la vinculación de individuos, grupos, comunidades políticas en unión duradera pero limitada de tal forma que permita la enérgica búsqueda de fines comunes a la vez que se mantiene la respectiva integridad de cada una de las partes (1987: 42)

En este caso, el principio federalista va más allá del ámbito de la política gubernamental y abarca diferentes instituciones y formas de organización de la sociedad. Nos encontramos ante un punto de vista muy enriquecedor, pues abre el camino para entender el principal fundamento del federalismo: combinar unidad y diversidad. Self-rule y shared rule es un principio que se aplica al estado federal. Desde esa perspectiva, Valdés (2012) afirma que el estado federal, en base a la teoría jurídico-política, se caracteriza por combinar unidad y diversidad y, al mismo tiempo, 
establecer un orden constitucional único para las acciones de self-rule y shared rule.

En lo que respecta al Estado federal, es conveniente reafirmar que no todos los sistemas federativos de gobierno se organizan con el mismo propósito y características. Esa es la discusión que Valdés (2012) presenta en su libro Los rostros del federalismo, animando a pensar sobre las particularidades que el sistema federativo de gobierno incorpora, dependiendo del país que lo adopte, en diferentes partes del mundo. En cuanto al origen, se encuentran en un primer grupo los federalismos cuyo objetivo original es alcanzar la unión en un Estado de entidades territoriales, anteriormente soberanas. En un segundo grupo están los federalismos cuya motivación es la descentralización del poder (Boisier, 1985; Díez, Gutiérrez y Pazzi, 2013). No obstante, independientemente de su origen, mantienen una característica común, a saber: "Los Estados federales actuales son, así, "antes que nada, Estados democráticos, que o —raramente- nacieron ya con tal carácter o —en la mayor parte de los casos - con el de Estados constitucionales en vías de democratización” (Valdés, 2012: 23, 24).

Considerando a este autor, se destaca que para que un Estado se mantenga como federación, no es necesaria sólo una Constitución, sino también una "cultura federal” (ídem.: 9), que a su vez se desdobla en actitudes sociales. Eso significa que "ausente esa cultura social, un Estado federal que ha nacido como tal, puede llegar a mantenerse o puede llegar a federalizarse uno cuyo punto de partida sea la centralización”. Un Estado federal solamente podrá mantenerse con un conjunto de hábitos, valores y comportamientos que sean federales. Por lo tanto, los elementos simbólicos están presentes en la estructura del federalismo y escapan a las normas y a las constituciones.

En Brasil, el régimen de gobierno federativo existe desde 1891 y para Abrucio (1998: 32) su origen estuvo relacionado con el "descontento ante el centralismo imperial”. Eso significa que las unidades federativas no estaban constituidas para definir la organización nacional, sino como forma de obtener "autonomía" e "independencia” del gobierno central. Otros autores tratan el origen distorsionado de la federación brasileña; por ejemplo, Castro (2005: 168) apunta que "la estructura federativa brasileña se estableció para acomodar [...] la perspectiva de manutención de una unidad que debería ser preservada a cualquier precio". Se trató de una acomodación del "mito de la unidad territorial" (Castro, 2005: 167, apud Machado, 1990), el cual, aún hoy, debilita el pacto federativo, buscando conciliar intereses entre las élites regionales y el gobierno central. Para Cataia (2001), la Federación surge de arriba hacia abajo, frente al poder del gobierno central, que dio el título de "estado" a las antiguas Provincias aunque, realmente, nunca hubo estados en Brasil que disfrutasen de soberanía, como sí ocurrió en Estados Unidos. Se trató de "un cambio de denominación: en 1891 las provincias pasaron a llamarse estados" (Cataia, 2001: 106). De esta forma, por causa de una construcción forzada del federalismo, desde 1891 Brasil acumula períodos de democracia y de autoritarismo. Se caracteriza, entonces, como una Federación inestable y dudosa, ya que no existe otro régimen que permita el federalismo, a menos que sea la democracia (Soares, 1998). Esa inestabilidad democrática pone en duda al federalismo brasileño. Así, interpretamos que la condición imprescindible del federalismo es un gobierno descentralizado y, por eso, si por un lado se considera que presentar la expresión fede- 
ralismo descentralizado es una redundancia, por otro, insistir en el término federalismo centralizado es una contradicción. Por causa de esos conflictos y los distintos contextos político-institucionales, la República brasileña alternó, en su trayectoria, el federalismo y el autoritarismo de la forma que se presenta en el Cuadro 1.

Cuadro 1. Centralización vs. descentralización: el péndulo del federalismo brasileño

\begin{tabular}{|l|l|}
\hline \multicolumn{1}{|c|}{ Periodo } & \multicolumn{1}{c|}{ Orientación federal } \\
\hline Primera República (1889-1930) & $\begin{array}{l}\text { Federalismo (Gran autonomía de los goberna- } \\
\text { dores de estado) }\end{array}$ \\
\hline Era Vargas (1930-1945) & Autoritarismo \\
\hline Segunda República (1945-1964) & Federalismo \\
\hline Período Militar (1964-1985) & Autoritarismo \\
\hline $\begin{array}{l}\text { Período de Redemocratización } \\
\text { (1988 hasta la actualidad) }\end{array}$ & Federalismo (Unidades federativas autónomas) \\
\hline
\end{tabular}

Fuente: Abrucio (1997). Elaborado por Vanessa da Silva Vieira.

Durante la Primera y la Segunda República, los gobiernos tenían un gran poder de decisión y durante el período en el que se intercaló la transición de la dictadura a la redemocratización, los gobernadores estatales tenían tanto poder de decisión que Abrucio (2005) los denominó “Barones de la Federación”. Realmente, eso no debería sorprender visto que una Federación está constituida por estados soberanos.

Durante la Era Vargas y la Dictadura Militar, la autonomía y la democracia se volvieron inoperantes sustituyendo el federalismo por autoritarismo. Valdés (2012: 24) afirma que "las dictaduras, con sus partidos únicos de gobierno y su negación de las elecciones libres, son incompatibles con la mecánica de funcionamiento del principio federal”. Entre 1964 y 1985, los planes elaborados por los estados federados estuvieron vinculados al proyecto de integración territorial de los gobiernos militares, a partir de la modernización tecnológica y la industrialización, sin considerar las especificidades de los estados y los municipios (Becker y Egler, 1993). Se debe Insistir que en ese período de régimen autoritario, Brasil y otros países de América Latina eran federativos, es un error, pues de acuerdo con Soares:

[...] em regimes autoritários não há, nem pode haver, pacto federal, porque a unidade está alicerçada em bases muito distintas: há um núcleo central de poder com capacidade de promover, via coerção, a união de territorialidade heterogênea. Evidentemente, este caminho dispensa as condições para o contrato federal, a unidade é imposta de cima para baixo e não envolve barganha [...] (1998: 148).

El proyecto de integración territorial elaborado y ejecutado por los militares, buscó mantener la autoridad política central, silenciando la expresión de los diversos intereses territoriales.

Desde el fin de la Dictadura Militar en 1985 hasta el año 1996, hubo un "modelo estadualista" (el "péndulo federativo favoreció a las unidades estatales en términos políticos y financieros”) y "predatorio" (el "comportamiento individualista" 
reforzó "prácticas salvajes de incentivo fiscal” que llevaron a nivel nacional a la “fragmentación del sistema político") (Abrucio, 1998). Aun así, desde una perspectiva espacial, se provocó una fragmentación territorial, mediante acciones desarticuladas y desorganizadas en las diferentes escalas gubernamentales.

En el período siguiente, el modelo federativo se debilitó debido a la disminución del poder de los estados cuyo marco legal fue la Enmienda Constitucional EC $n^{0} 15 / 1996$, que estableció nuevas reglas para la creación de municipios y la Ley Kandir (Ley Complementaria $\mathrm{n}^{\circ}$. 87/1996) que "reguló el ejercicio de sus competencias tributarias, de gasto y de implementación de políticas públicas” (Arretche, 2009: 378). Aunque esos marcos regulatorios hayan limitado el poder político y tributario de los gobiernos subnacionales, éstos poseen aún un importante margen de competencias. Desde el punto de vista geográfico, se destacan las siguientes: a) instaurar una regionalización adecuada a los objetivos y metas de cada gobierno; b) elaborar y ejecutar políticas territoriales ajustadas a las especialidades de sus respectivas escalas de actuación; c) captar recursos que contribuyan al buen desempeño del territorio mediante estrategias gubernamentales específicas. Esto significa que el modelo de planificación adoptado y los métodos utilizados para su ejecución se establecen de forma autónoma, mediante el aval de la Constitución. En este caso, optar por estrategias competitivas o cooperativas, con un bajo o un alto nivel de participación, que concentren o desconcentren inversiones, es una opción de cada gobierno en su escala de acción.

A partir de esas cuestiones, se ha optado por estudiar cómo se han comportado los gobernadores ante la autonomía que se les concedió, para elaborar y ejecutar estrategias que atiendan a las especificidades de sus territorios. En Bahía, por ejemplo, el poder ejecutivo representado por el gobernador del estado, utiliza frecuentemente los "incentivos fiscales" y los "incentivos territoriales" como estrategias para la promoción económica del territorio y utiliza los Planes Plurianuales para viabilizar tales estrategias (Vieira, 2009). Esta práctica influye en la gobernanza territorial y ayuda a entender los comportamientos de los gobiernos subnacionales, especialmente los gobiernos estatales, en ese "pacto federativo" (Abrucio, 1998, 1999, 2005; Rodrigues, 1998; Martin, 2005; Castro, 1997).

Obsérvese que en Brasil el fortalecimiento del pacto federativo, en cuanto a acción política y financiera coordinada entre los gobiernos subnacionales y el gobierno nacional, es tan necesario como conflictivo, debido a las particularidades socioespaciales construidas históricamente que guardan cada uno de sus 5.561 municipios y sus 27 unidades federativas. La diversidad favorece un ambiente político innovador, donde cada gobierno puede impulsar mecanismos y estrategias teniendo en cuenta las peculiaridades territoriales, los objetivos del plan gubernamental y el perfil político partidista. El Plan Plurianual es, en ese contexto, un instrumento para garantizar tales estrategias y mecanismos.

\section{Políticas territoriales en el contexto federativo: El Plan Plurianual (PPA)}

Conforme la describe Costa (2001), la territorial es un tipo de política pública “[...] formulada y aplicada lo más directamente posible, a las modificaciones en la estructura territorial del país", cuando se encuentran en el ámbito del gobierno fede- 
ral, pero también en la estructura territorial de estados y municipios, cuando las emprenden las respectivas escalas de los gobiernos subnacionales. Se trata de una regulación del territorio hecha por el Estado. Más allá de esta concepción, Silva y Fonseca (2008: 19) presentan una propuesta conceptual más amplia, a saber “[...] un conjunto de estrategias y acciones engendradas por varios agentes, en las más diversas escalas y sectores, con repercusiones directas en el espacio". Esta propuesta favorece los "mecanismos institucionales de regulación", incluyendo leyes, decretos, "planes y programas". Es por ello que se trata de una referencia importante en este trabajo, visto que las políticas territoriales se analizarán a partir de los Planes Plurianuales. El Plan Plurianual se ha convertido en el principal instrumento de planificación y presupuesto de las entidades federativas, conforme al art. 165 de la Constitución Federal de 1988, permitiendo a los gobiernos subnacionales la elaboración de un plan específico para sus respectivas escalas de acción:

Instrumento de planejamento e orçamento do governo federal que estabelecerá, de forma regionalizada, as diretrizes, objetivos e metas da administração pública federal para as despesas de capital e outras delas decorrentes e para as relativas aos programas de duração continuada (Brasil, 1988).

Aunque el texto constitucional no mencione a las unidades subnacionales, el PPA también es un instrumento de planificación de los estados y municipios. Excediendo su obligatoriedad constitucional y su función para la administración pública, el PPA representa para la Geografía política un instrumento de gestión, articulador de las estrategias espaciales mediante la delimitación de prioridades para el desarrollo del territorio, siendo por lo tanto un instrumento de base para la construcción del mapa de acciones desarrolladas por el gobierno, en términos de política territorial y de lectura geopolítica del espacio.

\subsection{Los Planes Plurianuales en Bahía}

Bahía, desde la Constitución de 1988, tuvo seis PPAs (Cuadro 2). Los cuatro primeros bajo la administración de un mismo grupo político, sometido a un modelo específico de gobierno (de derechas y clientelar), fundamentado en el "carlismo", corriente política liderada por el ex Senador Antônio Carlos Magalhães, y, los dos últimos elaborados por el gobierno de Jaques Wagner, del Partido de los Trabajadores (PT) (Vieira, 2014).

Cuadro 2. Planes Plurianuales de Bahía (periodos gubernamentales, cuatrienios y regionalización)

\begin{tabular}{|l|c|c|c|}
\hline \multicolumn{1}{|c|}{ Gobierno } & Periodo & PPA (4 años) & Regionalización \\
\hline Antonio Carlos Magalhães & $1991-1994$ & $1992-1995$ & - \\
\hline Paulo Souto & $1995-1998$ & $1996-1999$ & - \\
\hline César Borges & $1999-2002$ & $2000-2003$ & Eixos de desarrollo \\
\hline Paulo Souto & $2003-2006$ & $2004-2007$ & Eixos de desarrollo \\
\hline Jaques Wagner & $2007-2010$ & $2008-2011$ & Territorios de Identidad \\
\hline Jaques Wagner & $2011-2014$ & $2012-2015$ & Territorios de Identidad \\
\hline
\end{tabular}

Fuente: Planes Plurianuales de Bahía. Elaboración Vanessa da Silva Vieira. 
Los PPAs son elaborados por la Secretaría de Planificación (SEPLAN) y, siguiendo las directrices constitucionales, establecen una regionalización específica, definida particularmente por cada gobierno. Se desconoce la regionalización establecida para los dos primeros planes, pero se sabe que para los planes de los cuatrienios de 2000-2003 y 2004-2007, se instituyeron los Eixos ${ }^{3}$ de Desarrollo y, para los planes de los cuatrienios 2008-2011 y 2012-2015 los Territorios de Identidad.

Cuadro 3. Comparación de estructuras y conceptos del PPA 2004-2007 y PPA 2008-2011

\begin{tabular}{|c|c|c|}
\hline & PPA 2004-2007 & PPA 2008-2011 \\
\hline $\begin{array}{l}\text { Directrices } \\
\text { estratégicas }\end{array}$ & $\begin{array}{l}\text { Bahía de toda gente } \\
\text { Bahía que hace } \\
\text { Riquezas de la buena tierra } \\
\text { Caminos de Bahía } \\
\text { Gestión solidaria y gobierno } \\
\text { competente }\end{array}$ & $\begin{array}{l}\text { Directrices estrategias socioeco- } \\
\text { nómicas } \\
\text { Directrices Estratégicas territo- } \\
\text { riales } \\
\text { Directrices de implementación } \\
\text { de los principios de gestión }\end{array}$ \\
\hline Regionalización & 13 Eixos de Desarrollo & 26 Territorios de Identidad \\
\hline Evaluación & $\begin{array}{l}\text { Conjunto de actividades volcadas } \\
\text { a testar/medir la efectividad de } \\
\text { los programas y acciones, con } \\
\text { foco en el resultado; } \\
\text { Evalúa los impactos a partir de } \\
\text { los indicadores numéricos. }\end{array}$ & $\begin{array}{l}\text { Constitución de un Fórum de } \\
\text { Acompañamiento del PPA for- } \\
\text { mado por representantes de los } \\
\text { territorios de identidad y técnicos } \\
\text { del gobierno, para el control } \\
\text { social de las políticas públicas, a } \\
\text { partir del monitorización y eva- } \\
\text { luación }\end{array}$ \\
\hline Participación & $\begin{array}{l}500 \text { representantes seleccionados } \\
\text { de los segmentos sociales orga- } \\
\text { nizados participaron de las con- } \\
\text { sultas durante } 30 \text { días de reunio- } \\
\text { nes. No existe especificación del } \\
\text { origen de los representantes. }\end{array}$ & $\begin{array}{l}12.246 \text { representantes de los } \\
\text { diferentes sectores sociales de } \\
\text { cada uno de los } 26 \text { territorios de } \\
\text { identidad se reunieron en plenos } \\
\text { durante un período aproximado } \\
\text { de } 30 \text { días. }\end{array}$ \\
\hline $\begin{array}{l}\text { Colaboración } \\
\text { entre las institu- } \\
\text { ciones y entida- } \\
\text { des }\end{array}$ & $\begin{array}{l}\text { Principio de la Colaboración- } \\
\text { Público-Privada como fuente de } \\
\text { financiamiento de los recursos } \\
\text { públicos }\end{array}$ & $\begin{array}{l}\text { Representantes de los territorios } \\
\text { de identidad / Representantes de } \\
\text { los movimientos sociales / Uni- } \\
\text { versidades / Sector empresarial / } \\
\text { Instituciones de investigación } \\
\text { científica y tecnológica }\end{array}$ \\
\hline
\end{tabular}

Fuente: PPA 2004-2007 y PPA 2008-2011. Elaboración: Vanessa da Silva Vieira

Con el objetivo de establecer una perspectiva comparada sobre las prioridades espaciales que orientaron al PPA en cada gobierno, se han seleccionado dos, a saber: el PPA 2004-2007 y el PPA 2008-2012. La intención es destacar sus motivaciones específicas para establecer una regionalización, pues se defiende que la

3 Eixo: término asociado a una línea recta imaginaria dentro del territorio bahiano, que representa las principales vías de circulación de dinero, mercadurías y personas. 
misma es reflejo de la concepción de desarrollo territorial que cada gobierno posee internamente. De esta forma, más adelante se presentarán las motivaciones que estos gobiernos revelaron al tratar sus estrategias espaciales. Sin duda, en ambos períodos los PPA constituyen un eje de intervención política considerado prioritario, al que se destinaron 13.904 millones de reales en 2004-2007 (de un total de los cuatro presupuestos acumulados de 62.202 correspondientes al Estado de Bahia), cifra que se incrementó a 18.947 millones de reales en 2008-2011 (sobre un total también mayor de 93.037 de los cuatro años sumados). Por lo tanto, el dinero público consignado en los dos períodos a los PPAs ha supuesto de forma continuada más del 20\% del presupuesto anual total de Estado.

En términos generales, ambos PPAs incluyen puntos exigidos constitucionalmente, es decir, regionalización, evaluación, participación y colaboración entre las instituciones y entidades. Pero, conforme al Cuadro 3, el modelo de tratamiento de estos puntos es, como mínimo en términos oficiales, distinto.

\subsection{El PPA 2004-2007}

El PPA 2004-2007 fue elaborado por el gobierno de Paulo Souto (2003-2006) y la regionalización establecida pretende atender al estatus de competitividad de la economía global, favoreciendo como estrategia a los grandes corredores de circulación de la producción (Vieira, 2009). En realidad, los Eixos de Pensamiento de Desarrollo, Figura 1, son los grandes ejes de la circulación del capital.

Figura 1. Regionalización para la implantación de los programas del PPA 2004-2007

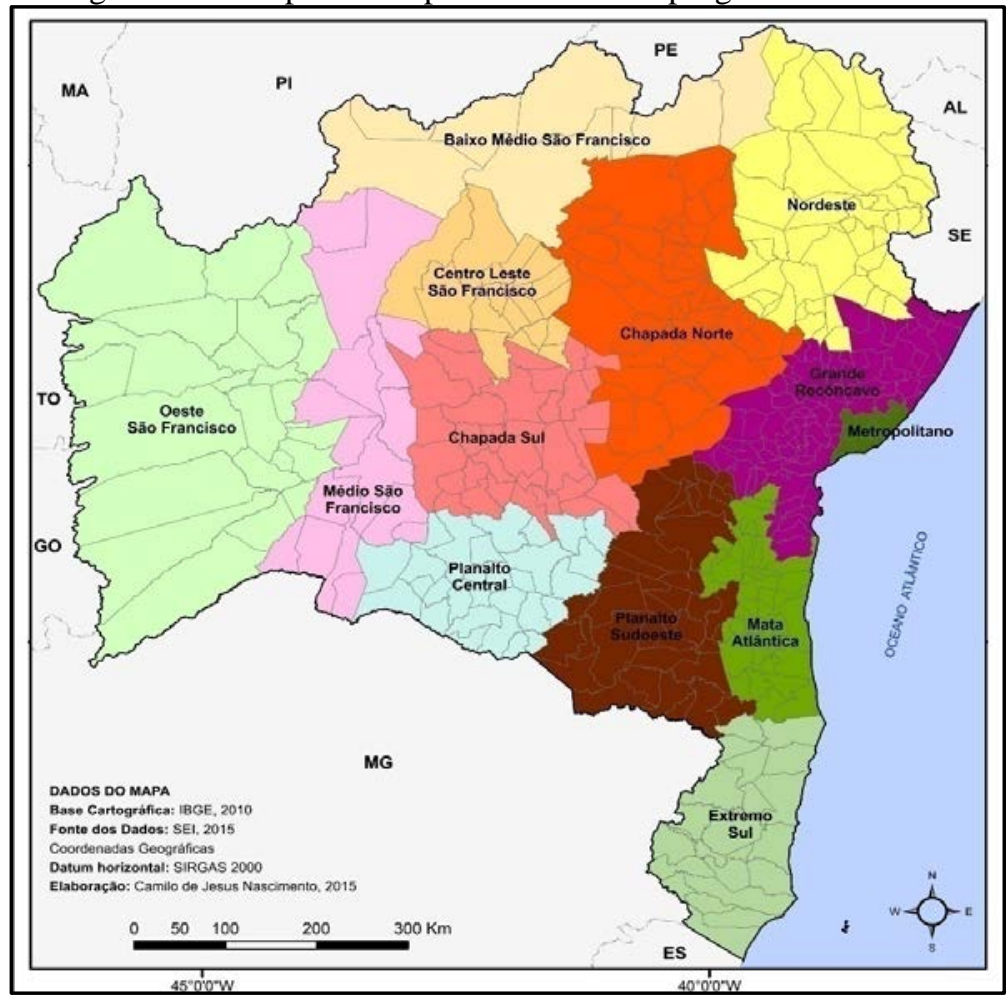


Desde el punto de vista oficial, los Eixos de Desarrollo se agruparon a partir del grado de dinamismo y de infraestructura. De esta forma, tal y como se presenta en el Cuadro 4, se determinan los eixos dinámicos y dotados de competitividad, localizados en la parte costera y occidental respectivamente.

Cuadro 4. Clasificación y caracterización de las Eixos de Desarrollo según

el PPA 2004-2007

\begin{tabular}{|c|c|c|c|}
\hline Eixos de Desarrollo & Localización & Actividades & $\begin{array}{c}\text { Infraestructura y } \\
\text { Competitividad }\end{array}$ \\
\hline $\begin{array}{l}\text { Metropolitano, Gran } \\
\text { Recôncavo, Mata } \\
\text { Atlântica y Extremo } \\
\text { Sur }\end{array}$ & Parte costera & $\begin{array}{l}\text { Inversiones turísticas } \\
\text { nacionales e internacio- } \\
\text { nales. } \\
\text { Emprendimientos in- } \\
\text { dustriales. } \\
\text { Actividades importan- } \\
\text { tes de comercio y servi- } \\
\text { cios concentrados. }\end{array}$ & $\begin{array}{l}\text { Disponen de las mejo- } \\
\text { res condiciones de } \\
\text { infraestructura del esta- } \\
\text { do. } \\
\text { Dinámica y competitiva }\end{array}$ \\
\hline $\begin{array}{l}\text { Bajo Medio São } \\
\text { Francisco, Centro } \\
\text { Este São Francisco, } \\
\text { Medio São Francis- } \\
\text { co y Oeste de São } \\
\text { Francisco }\end{array}$ & $\begin{array}{l}\text { Parte occi- } \\
\text { dental }\end{array}$ & $\begin{array}{l}\text { Expansión agroindus- } \\
\text { trial en formas moder- } \\
\text { nos. }\end{array}$ & $\begin{array}{l}\text { Dinámica y competiti- } \\
\text { va. } \\
\text { Necesita infraestructu- } \\
\text { ra, sobre todo logística } \\
\text { de transporte local y } \\
\text { encaminamiento de la } \\
\text { producción. }\end{array}$ \\
\hline $\begin{array}{l}\text { Nordeste, Chapada } \\
\text { Norte, Chapada Sur, } \\
\text { Planalto Sudoeste y } \\
\text { Planalto Central }\end{array}$ & $\begin{array}{l}\text { Parte cen- } \\
\text { tral; } \\
\text { Semiárido }\end{array}$ & $\begin{array}{l}\text { Explotación de cultivos } \\
\text { tradicionales y de sub- } \\
\text { sistencia. } \\
\text { Algunas actividades de } \\
\text { minería. }\end{array}$ & $\begin{array}{l}\text { No disponen de compe- } \\
\text { titividad en el plano } \\
\text { económico. } \\
\text { Bajos indicadores so- } \\
\text { ciales y de infraestruc- } \\
\text { tura. }\end{array}$ \\
\hline
\end{tabular}

Fuente: Plan Plurianual 2004-2007. Elaboración Vanessa da Silva Vieira.

También hay un foco sin competitividad, localizado en la parte central del estado:

A abertura das fronteiras nacionais para a produção e comercialização em escala mundial tem contribuído para adensar caminhos e criar outros novos, articulando os fluxos inter-regionais aos fluxos locais. Tal estratégia tem levado as políticas públicas e privadas a adotarem critérios espaciais de investimentos, destacandose os grandes eixos de circulação como promotores e irradiadores espaciais do desenvolvimento. Dessa forma, a adoção de qualquer política no âmbito estadual não pode deixar de contemplar esses critérios de espacialidade, especialmente quanto aos eixos de circulação e de transporte (Bahia, 2003: 20-21). 
Para que tales propósitos fuesen cumplidos, se vincularos a las Eixos de Desarrollo, 13 Ciudades Estratégicas, que ejercieron el papel de soporte urbano en el desarrollo regional, actuando como agentes de fortalecimiento de las Eixos de Desarrollo.

En este caso y, de acuerdo con Silva (2006), la implementación del mencionado programa favoreció a la maximización de los equilibrios inter-regionales, ya que potencialmente, se impulsaba la concentración de inversiones en las ciudades más dinámicas de los focos, sin estimular a otras ciudades poseedoras de una diversidad de condiciones. Desde el punto de vista gubernamental, tales urbes estratégicas son los núcleos de una red que favorecen la circulación del capital. Fueron 30 ciudades que tuvieron la función de propagar el desarrollo ipara un total de 417! El Cuadro 5 muestra la distribución de las ciudades estratégicas dentro de las trece Eixos de Desarrollo.

Cuadro 5. Distribución de las ciudades estratégicas por las Eixos de Desarrollo

\begin{tabular}{|l|c|c|l|}
\hline \multirow{2}{*}{ Eixos de Desarrollo } & Número de & \multicolumn{2}{|c|}{ Ciudades estratégicas } \\
\cline { 3 - 5 } municipios & Núm. & \multicolumn{1}{|c|}{ Nombre } \\
\hline Metropolitano & 10 & - & \\
\hline Gran Recôncavo & 78 & 4 & $\begin{array}{l}\text { Feira de Santana, Santo Antonio de } \\
\text { Jesus, Alagoinhas, Valença }\end{array}$ \\
\hline Mata Atlântica & 45 & 2 & Itabuna, Ilhéus \\
\hline Extremo Sur & 21 & 2 & Texeira de Freitas, Eunapólis \\
\hline Nordeste & 47 & 3 & $\begin{array}{l}\text { Euclides da Cunha, Paulo Afonso, } \\
\text { Ribeira do Pombal }\end{array}$ \\
\hline Chapada Norte & 49 & 4 & $\begin{array}{l}\text { Ipirá, Itaberaba, Jacobina, Senhor } \\
\text { do Bomfim }\end{array}$ \\
\hline Chapada Sur & 33 & 1 & Seabra \\
\hline Planalto Sudoeste & 39 & 3 & $\begin{array}{l}\text { Jequié, Vitória da Conquista, Itape- } \\
\text { tinga }\end{array}$ \\
\hline Planalto Central & 29 & 2 & Brumado, Guanambim \\
\hline Bajo Medio São Francisco & 8 & 1 & Juazeiro \\
\hline Centro Este São Francisco & 19 & 1 & \begin{tabular}{l} 
Irecê \\
\hline Medio São Francisco
\end{tabular} \\
\hline Oeste São Francisco & 23 & 2 & $\begin{array}{l}\text { Bom Jesus da Lapa, Ibotirama, } \\
\text { Barra }\end{array}$ \\
\hline
\end{tabular}

Fuente: Plan Plurianual 2004-2007. Elaboración Vanessa da Silva Vieira.

Si analizamos de forma general como se distribuyeron los fondos públicos destinados a estos Eixos de Desarrollo tomando como ejemplo el año 2004 observa- 
mos que la mayor parte de los mismos se dirigieron a intervenciones que concernían a todo el Estado (3.954 millones de Reales, el 29,04\% del total), mientras que el territorio metropolitano recibió el 10,94\% y el Grande Recóncavo próximo el 4,41\%, no dirigiéndose más del 3,5\% a ninguno de los restantes territorios establecidos, según datos del propio Governo da Bahia.

En este contexto, prevalecen la división, selección y concentración de los recursos financieros en determinadas localidades, surgiendo subespacios dotados de infraestructuras tecnológicas y modernas, es decir, focos de dinamismo que se diferencian profundamente de su entorno, como por ejemplo, las ciudades de Feira de Santana, Juazeiro, Vitória da Conquista, Eunápolis, entre otras. En contrapartida, las ciudades de su entorno, tales como las que están en los Eixos "que no mostraban competitividad”, no se beneficiaron de las políticas de infraestructura.

La estrategia de desarrollo utilizada para consolidar los grandes eixos de circulación, a partir del uso de incentivos fiscales y, también, de la colaboración público-privada, aseguró la implantación de industrias en el interior del estado, fortaleciendo la base industrial de una serie de ciudades medias. Pero, por otro lado, dichas estrategias no consiguieron reducir los desequilibrios regionales, resolver el “enigma bahiano" de la tenue integración urbano-regional, ni dinamizar centros urbanos en las regiones más vulnerables socioeconómicamente, localizadas en el semiárido (Silva, 2008).

Se aplicó una acción polarizadora de las ciudades más desarrolladas acentuando, por tanto, las islas de crecimiento económico. Para tener una idea de esa concentración, a partir de los datos del IBGE se observa que en Bahía 25 ciudades concentran el 75\% del Producto Interior Bruto (PIB) estatal y, ¡tan solo 5 ciudades retienen 50\% del PIB! (Silva, 2006). La concentración de esfuerzos en los grandes corredores de circulación, en las ciudades estratégicas y en los grandes emprendimientos, tomando como telón de fondo los grandes eixos de desarrollo, evidenció una política selectiva e institucional que reforzó la selectividad económica y corporativa.

\subsection{EI PPA 2008-2011}

En el Plan Plurianual (2008-2011) elaborado por el Gobierno de Jaques Wagner (2007-2010) se definió como unidad regional 26 Territorios de Identidad (Figura 2), concebidos, desde el punto de vista oficial, como:

[...] é um espaço físico, geograficamente definido, geralmente contínuo, caracterizado por critérios multidimensionais, tais como o ambiente, a economia, a sociedade, a cultura, a política e as instituições, ocupado por uma população com grupos sociais relativamente distintos, que se relacionam interna e externamente, por meio de processos específicos, onde se pode distinguir um ou mais elementos que indicam identidade e coesão social, cultural e territorial (Bahia, 2008: 58). 
Figura 2. Regionalización para la implantación de los programas en el PPA 2008-2011

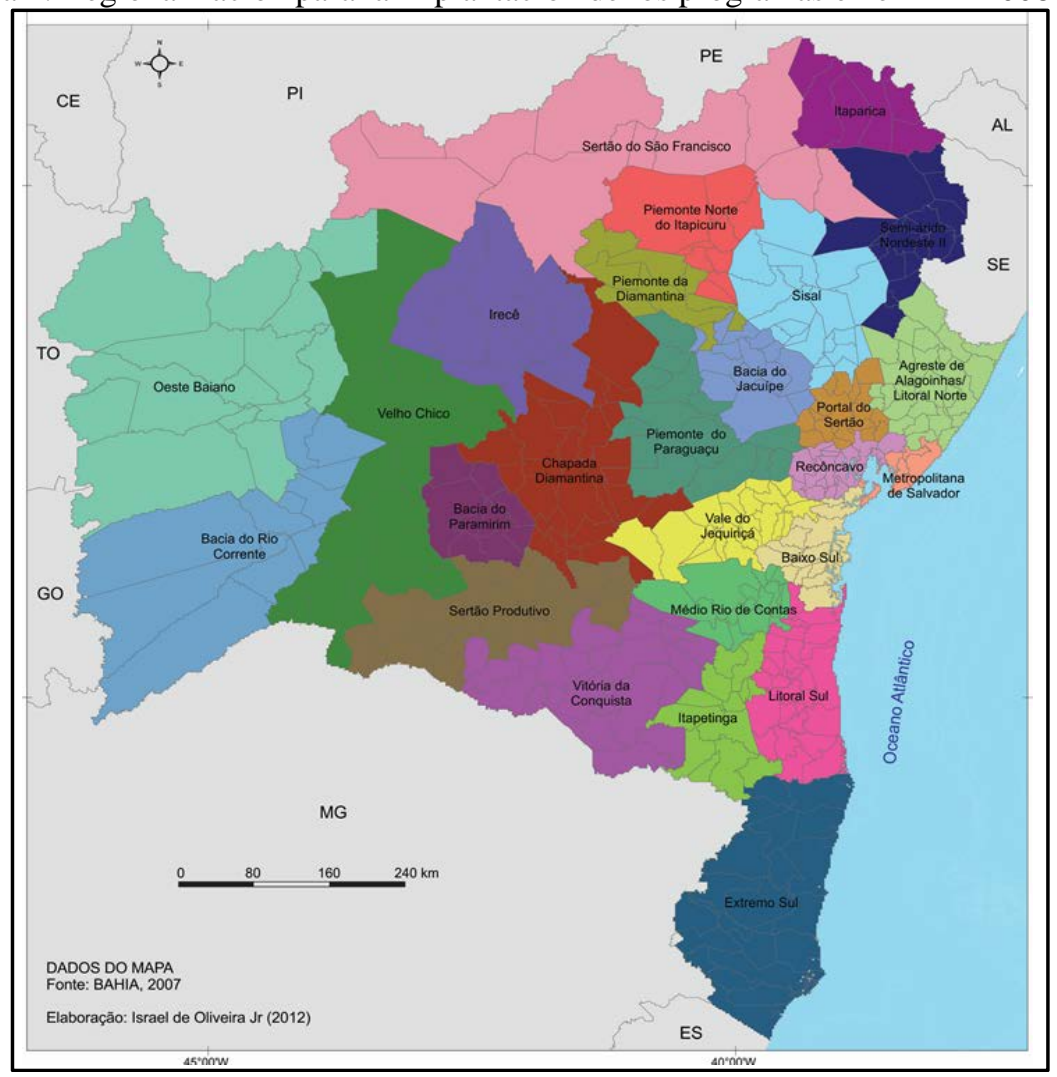

Esta explicación conceptual es la misma que la formulada por el Ministerio de Desenvolvemento Agrario. Estamos ante una explicación amplia y confusa en relación a los términos “territorio" e "identidad”, puesto que no es posible identificar las territorialidades impulsoras para la construcción de tales demarcaciones, ni tampoco los criterios que definen la identidad de los municipios para componer las unidades de planificación; de hecho, cabe destacar que en ocasiones hubo una mera agrupación de municipios. Esta práctica dificultó la minimización de las desigualdades socioeconómicas y la inclusión socioterritorial, principalmente debido a las especificidades que se mantuvieron en cada delimitación de base municipal.

Según consta en el referido plan, la inspiración para la creación de los Territorios de Identidad surgió a partir de los Territorios Rurales que consisten en una delimitación espacial para la acción del gobierno federal. Esa regionalización surgió de la Coordinación de los Territorios y Movimientos de Agricultura y del Ministerio de Desarrollo Agrario. En relación a ello, el Gobierno de Jaques Wagner llevó a cabo algunos ajustes conceptuales y metodológicos, instituyendo a los Territorios de Identidad como unidades de planificación. De acuerdo con la Secretaría de Planificación de Bahía, la finalidad de los Territorios de Identidades, en aquel momento, era "identificar oportunidades de investimento e prioridades temáticas definidas a partir da realidade local de cada Território, possibilitando o desenvolvimento equilibrado e sustentável entre as regiões [...]” (Bahia, 2008). 
En términos oficiales, el objetivo fue el de convertir a los territorios en protagonistas del propio desarrollo a partir de la puesta en valor de las potencialidades endógenas. Eso significa que la capacidad de desarrollo del Territorio de Identidad debería constituir la base de una nueva relación entre los actores locales y el gobierno. Se trataba de una propuesta de modelo de gestión que traía consigo un cambio estructural, pues pretendía construir una relación a partir de la organización local y la formalización de acuerdos de cohesión social que favoreciese el desarrollo de las potencialidades locales y, no simplemente, la transferencia de recursos orientados a acciones desvinculadas de las características de dichos territorios. En el intento de alcanzar esos objetivos, se creó un "Fórum de Acompañamiento del PPA", con representantes de cada uno de los Territorios de Identidad. La gran diferencia de ese plan fue considerar la efectiva participación de la sociedad civil en la elaboración, ejecución y evaluación, lo que significó una nueva propuesta para la planificación del estado de Bahía. En la fase de elaboración, por ejemplo, se realizaron plenos en cada uno de los Territorios de Identidad, donde representantes de la población indicaban las necesidades más latentes de su espacio particular que deberían constar, en forma de programas, en el Plan Plurianual. Se trataba de saber, por ejemplo, hasta qué punto esas necesidades indicadas por las plenarias fueron convertidas en programas y transformadas en acciones. Estamos ante un aspecto que solamente una investigación más profunda podría responder, porque no se encontraron instrumentos institucionalizados capaces de asegurar de forma efectiva y eficaz esa nueva propuesta.

La pauta gubernamental privilegió espacialmente al semiárido debido a su extensión en Bahía. De los 26 Territorios de Identidad, 18 estaban completa o parcialmente situados en el semiárido, a saber: Velho Chico, Irecê, Bacia do Paramirim, Sertão Produtivo, Vitória da Coquista, Itapetinga, Médio Rio de Contas, Vale do Jequiriça, Portal do Sertão, Bacia do Jacuípe, Sisal, Piemonte Norte do Itapicuru, Piemonte da Diamantina, Chapada Diamantina, Semiárido Nordeste, Itaparica, Sertão do São Francisco, Agreste de Alagoinhas. Esos Territorios de Identidades fueron contemplados con programas y acciones que consideraron sus limitaciones, sobre todo aquellas ligadas a cuestiones hídricas y, también, las relacionadas con la puesta en valor sus potencialidades, incluyendo la producción familiar y orgánica.

El impulso a las potencialidades del semiárido no significó dejar de lado el "resto del territorio", sino que cuando menos se utilizó para atenuar el discurso tradicional e imperativo enunciado por las élites regionales sobre el hecho de que el semiárido era el "resto del territorio", desde los puntos de vista infraestructural, social y económico. Se defiende que los planes gubernamentales tienen un papel importante en la superación de este discurso, en la medida en que establecen programas y acciones más solidarias, convocando a la sociedad civil para participar en la construcción de organizaciones sociales institucionales adecuadas para la formalización de un desarrollo territorial integrado. Estos planes procuran generar políticas innovadoras que contemplen las limitaciones hídricas y demuestran que hay "espacio" para construir nuevas territorialidades.

Asimismo, desde el punto de vista de la estrategia espacial, el territorio bahiano se dividió en cuatro Directrices Estratégicas Territoriales (D.E.T.). El Cuadro 6 muestra cada una de esos Eixos y las prioridades gubernamentales. 
Cuadro 6. Directrices Estratégicas Territoriales del PPA 2008-2011

\begin{tabular}{|c|c|c|}
\hline D.E.T. & Acciones & Caracterización \\
\hline $\begin{array}{l}\text { Desarrollar } \\
\text { el semiárido } \\
\text { bahiano }\end{array}$ & $\begin{array}{l}\text { Promoción de la organización socio-productiva con } \\
\text { oferta hídrica para consumo humano y de la produc- } \\
\text { ción; apoyo al productor con oferta de crédito y forta- } \\
\text { lecimiento de las cadenas productivas; estímulo a la } \\
\text { implantación de unidades de beneficios de la produc- } \\
\text { ción y de emprendimientos no-agrícolas; fomento de } \\
\text { la investigación, difusión de conocimientos, de in- } \\
\text { formaciones y de tecnologías adaptadas al semiárido, } \\
\text { articulando aquellas acciones que garanticen la sus- } \\
\text { tentabilidad ambiental. }\end{array}$ & $\begin{array}{l}\text { Extrema pobre- } \\
\text { za y desigualdad } \\
\text { socioeconómi- } \\
\text { ca; heterogénea } \\
\text { y dotada de } \\
\text { necesidades y } \\
\text { potencialidades } \\
\text { específicas. }\end{array}$ \\
\hline $\begin{array}{l}\text { Consolidar } \\
\text { la Región } \\
\text { Metropolita- } \\
\text { na de Salva- } \\
\text { dor (RMS) } \\
\text { como polo } \\
\text { metropoli- } \\
\text { tano de ser- } \\
\text { vicios }\end{array}$ & $\begin{array}{l}\text { Cualificación de los servicios de hotelería, gastrono- } \\
\text { mía, salud, entretenimiento, educación, cultura, re- } \\
\text { ceptividad y creación de rutas turísticas; explotación } \\
\text { del potencial de Bahía de Todos los Santos para la } \\
\text { navegación, pesca con caña y submarina, deportes } \\
\text { náuticos e industria náutica, de entre otras activida- } \\
\text { des; desarrollo y fortalecimiento de los servicios que } \\
\text { complementan la actividad empresarial }\end{array}$ & $\begin{array}{l}\text { Por el prestador } \\
\text { de servicios }\end{array}$ \\
\hline $\begin{array}{l}\text { Dinamizar la } \\
\text { región del } \\
\text { cacao }\end{array}$ & $\begin{array}{l}\text { Retomar la producción del cacao, diversificar las } \\
\text { actividades productivas, apoyar a la expansión de } \\
\text { agroindustrias y atracción de nuevos emprendimien- } \\
\text { tos para la región; disponer asistencia técnica, crédito } \\
\text { e investigación agrícola, procurando agregar valores } \\
\text { a los productos; invertir en obras de infraestructura y } \\
\text { apoyar el proceso de renegociación de las deudas de } \\
\text { los productores rurales. }\end{array}$ & \\
\hline $\begin{array}{l}\text { Dinamizar } \\
\text { las diferen- } \\
\text { tes regiones } \\
\text { del estado de } \\
\text { Bahía }\end{array}$ & $\begin{array}{l}\text { Dotación de infraestructuras sociales y económicas; } \\
\text { identificación y fomento de sus potencialidades eco- } \\
\text { nómicas. Se incluyen aquí el Litoral Norte, Bajo Sur, } \\
\text { Extremo Sur y el Oeste Bahiano }\end{array}$ & $\begin{array}{l}\text { Demás subespa- } \\
\text { cios estatales } \\
\text { que constituirán } \\
\text { objetos de inter- } \\
\text { vención del } \\
\text { estado. }\end{array}$ \\
\hline
\end{tabular}

Fuente: PPA 2008-2011. Elaboración Vanessa da Silva Vieira.

El trato puntual que se dio a las regiones descritas en el Cuadro 6, se debe a los problemas específicos que existían entonces (y que aún existen). Para este caso, en el documento adoptado se encuentran algunas motivaciones para tal trato. Desde el punto de vista oficial, el semiárido concentra cerca del 50\% de la población de todo el estado y los peores indicadores sociales se suman a una estructura agraria concentrada. Se partió del principio de que para atenuar esta situación, era necesaria una política de uso y captación de recursos hídricos adecuados a la realidad y, también, incentivar a las organizaciones sociales productivas capaces de alterar positivamente la situación socioeconómica. En el caso de la Región Metropolitana de Salvador se valoró su condición de polo prestador de servicios y su potencial para convertirse en un espacio privilegiado de articulación de la economía de un sector 
del continente sudamericano con el mercado global. Y, en relación a la región del cacao, se partió de la crisis socioeconómica relacionada con las labranzas de cacao, devastada por el hongo Crinipellis perniciosa; así, se interpretó que existía una demanda urgente de diversificación de la base productiva y dirigida a la renegociación de las deudas de los cultivadores de cacao.

De hecho, un análisis de la distribución territorializada de los Presupuestos del Estado correspondientes a 2008, ya nos muestra que la mayor parte de los recursos se destinó s los programas que se dirigían al conjunto de Bahia (14.124.000 Reales, que suponían el 74,55\%), mientras que el área metropolitana de Salvador retrocedía hasta el 10,02\%, distribuyéndose el resto del gasto de forma bastante equitativa en intervenciones concretas e cada uno de los Territorios de Identidad creados.

El gobierno de Jaques Wagner, a partir del PPA 2008-2011, diseñó una política de intervención territorial en Bahía, añadiendo aspectos físicos relacionados con la materialidad del territorio, dotó de infraestructura técnica a las diferentes unidades regionales, pero también se trató de actuar a partir de una concepción simbólica del territorio que, aunque necesite precisiones, se abrió camino gracias a la planificación gubernamental que tenía en cuenta lo inmaterial, la "identidad”.

\section{Consideraciones finales}

El análisis del federalismo desde la geografía concede al territorio una centralidad no observada anteriormente. De esta forma, el territorio no es tan sólo un escenario de acción gubernamental, sino que también constituye la propia materia prima para la elaboración de las políticas, frente al uso de un conjunto de normas aprobadas en el sistema de gobierno federativo.

En Brasil, la Constitución Federal de 1998 representa un punto de ruptura en la redefinición de las reglas de juego político, ya que la democratización permitió en términos político-administrativos nuevas prácticas de planificación. De hecho, en la esfera de los gobiernos estatales se generan acciones, vinculadas a las normas federales. No obstante, es innegable que dichos gobiernos consiguieron un mayor grado de autonomía y, con eso, incluyeron en su planificación estrategias específicas de desarrollo territorial condicionadas por las diferentes posiciones políticas asumidas.

En nuestro caso, el Plan Plurianual se presentó en Bahia como un instrumento importante para la acción gubernamental. La obligación que tienen los gobiernos de institucionalizar las regiones para llevar a cabo las directrices y programas, va más allá de la exigencia burocrática. Se trata, en una interpretación geográfica, de una forma de control sobre la diversidad territorial, en coherencia con los objetivos particulares de casa gobierno. Tal control permite el uso y valorización de las potencias económicas presentes en la propia diversidad territorial. Está probado que las estrategias para el uso de dichas potencias puede tanto inducir a la integración, como atenuar la fragmentación del territorio.

Guiándose por la cuestión central esbozada en este artículo: ¿De qué forma se han comportado los gobernadores ante la autonomía que les fue concedida para elaborar y ejecutar estrategias que atiendan las especificidades de su territorio? A priori, su forma de actuar en el territorio depende directamente de la concreción de 
una agenda de gobierno, definida a partir de un programa político, en la que se determinan particularmente las directrices, las metas y los programas. Se trata así de optar por una opción seria y de decidirse por estrategias competitivas que contemplen un desarrollo equitativo del territorio, inclusivo y más permeable a la participación de la población. Sin duda, se trata de una lógica repetida en el marco de las políticas públicas, donde programa, creación de una nueva agenda y acción siguen una secuencia más o menos convencional. Todo esto sin despreciar una lectura geopolítica de la realidad, pues los gobiernos privilegian aquellos lugares, comunidades o territorios asociados a una iniciativa clara a desarrollar y donde cuentan con apoyos explícitos, que se pueden traducir en votos electorales.

En lo que respecta al Gobierno de Paulo Souto, la regionalización que dirigió los programas del Plan Plurianual, privilegió los espacios más competitivos desde el punto de vista de la globalización y modernización tecnológicas. En este caso, el objetivo fue el de establecer Bahía como un núcleo en el circuito global de la producción, mediante la transformación de la base económica y la captación de inversiones internacionales a cualquier precio. Como se ha apuntado, se trata de la trasposición a Bahia de los clásicos programas de creación de polos industriales y de desarrollo, ciudades de equilibrio, que a partir de su dinamismo económico y social consigan transferir a sus áreas de influencia los beneficios del crecimiento productivo y de la modernización.

En cuanto al Gobierno de Jaques Wagner, la novedad es el trato dado al territorio semiárido. Al menos en términos propositivos, ese espacio fue insertado en la agenda política del gobierno bahiano como prioridad absoluta. De manera nítida se reconoció que el semiárido no es un problema climático, sino un problema social. Para el ámbito estatal analizado necesita de políticas territoriales que valoren su potencial. En este caso, las lógicas de discriminación positiva y de justicia espacial se convirtieron en guías de las políticas implementadas, suponiendo un traslado al territorio de las lógicas de igualdad y reequilibrio social que contienen muchos programas políticos de la izquierda.

Como se comprueba, un análisis del Plan Plurianual permite identificar el perfil de gestión, las prioridades territoriales, las metas de un gobierno y la lectura geopolítica del territorio. Pero tan sólo una investigación más profunda, que se proponga analizar los diferentes momentos de la política pública, desde su elaboración hasta su implantación, podrá revelar si de verdad los impactos proyectados en la fase de formulación corresponden a los impactos reales. Ese es el camino que se pretende seguir en el futuro.

\section{Bibliografía}

Abrucio, Fernando Luiz (1998) Os barões da Federação: os governadores e a redemocratização brasileira. São Paulo: Hucitec/USP.

Abrucio, Fernando Luiz (1994) “Os barões da federação”. Lua Nova, núm. 33, 165-183.

Abrucio, Fernando Luiz (1999) "O longo caminho das reformas dos governos estaduais: crise, mudanças e impasses”, en M. A. Melo (ed.) Reforma do Estado e mudança institucional no Brasil. Recife: Fundação Joaquim Nabuco/Massangana, 161-198. 
Abrucio, Luiz Fernando (2005) "Reforma do Estado no federalismo brasileiro: a situação das administrações públicas estaduais”. Revista de Administração Pública (Rio de Janeiro), vol. 39, núm. 2, 401-420.

Abrucio, Fernando Luiz, y Samuels, David (1997) “A nova política dos governadores”. Revista de Cultura e Política, núm. 40/41, 137-166.

Agnew, J. (1987) Place and Politics. The Geographical Mediation os State and Society. Boston: Allen \& Unwin

Andrade, Manuel Correia de, y Andrade, Sandra Maria Correia de (1999) A federação brasileira: aspectos geo-políticos e geográficos. Contexto: São Paulo.

Araujo, Alex Macedo de (2009) Dinâmica do federalismo brasileiro e guerra fiscal. Disertación de Mestrado em Geografia. Pós-graduação da Faculdade de Filosofia, Letras e Ciências Humanas, Universidade de São Paulo, São Paulo.

Arretche, Marta (2009) “Continuidade e descontinuidades da Federação Brasileira: de como 1988 facilitou”. DADOS - Revista de Ciências Sociais (Rio de Janeiro), vol. 52, núm. 2, 377-423.

Arretche, Marta (2010) “Federalismo e igualdade territorial: uma contradição em termos?”. DADOS - Revista de Ciências Sociais (Rio de Janeiro), vol. 53, núm.3, 587-620.

Bahia (2003) Plano Plurianual 2004-2007. Salvador: Seplantec.

Bahia (2007) Plano Plurianual 2008-2011. Salvador: Seplan.

Becker, Bertha, K., y Egler, Cláudio (1993) BRASIL: Uma nova potência regional na economia-Mundo. São Paulo: Bertrand Brasil.

Boisier, Sergio (1985) "Un difícil equilibrio: centralización y descentralización en la planeación regional”. Revista El Trimestre Económico, vol. 52, núm. 205, 195-224.

Brasil (1988). Constituição da República Federativa do Brasil de 1988. Rio de Janeiro: Bloch editores.

Castillo, Ricardo; Toledo Júnior, Rubens de, y Andrade, Júlia (1997) “Três dimensões da solidariedade em Geografia. Autonomia político-territorial e tributação”. Revista Experimental (São Paulo: LABOPLAN - DG/FFLCH/USP), vol. II, núm. 3, 69-99.

Castro, Iná Elias de (1997) "Solidariedade territorial e representação. Novas questões para o pacto federativo nacional”. Revista Território, vol. 1, núm. 2, 33-42.

Castro, Iná Elias de (2005) Geografia e Política: território, escalas de ação e instituições. Rio de Janeiro: Bertrand do Brasil.

Castro, Iná Elias de (2007) “Décentralisation, démocratie et représentation législative locale au Brésil”. L’Espace Politique, núm. 3, 60-73.

Cataia, Márcio (2001) “Território Nacional e Fronteiras Internas. A fragmentação do território brasileiro”. Tesis de Doutorado em Geografia. Faculdade de Filosofia, Letras e Ciências Humanas, Universidade de São Paulo, São Paulo.

Claval, Paul (1978) Espacio y Poder. México: Fondo de Cultura Económica.

Costa, Wanderley Messias da (2001) O Estado e as políticas territoriais no Brasil. São Paulo: Contexto.

Dallabrida, Valdir Roque, y Zimmermann, Viro (2009) “Descentralização na Gestão Pública e Estruturas Subnacionais de Gestão do Desenvolvimento: o papel dos Consórcios Intermunicipais”. Revista Brasileira de Gestão e Desenvolvimento Regional, núm. 5, 328.

Díez, José Ignacio; Gutiérrez, Ricardo Raúl, y Pazzi, Andrés (2013): “¿De arriba hacia abajo o de abajo hacia arriba? Un análisis crítico de la planificación al desarrollo en 
América Latina”. Revista Geopolítica(s). Revista de estudios sobre espacio y poder, vol. 4, núm. 2, 199-235.

Egler, Cláudio, y Mattos, Margarida (2003) "Federalismo e gestão do território: as regiões integradas de desenvolvimento", en Anais do Encontro Nacional da Associação Nacional de Pós-graduação e Pesquisa em Geografia (ENANPEGE) (vol. 5). Florianópolis: UFSC, 426-434.

Eleazar, Daniel (1987) Exploring the federalismo. Tuscaloosa (Alabama): The University of Alabama Press.

Fonseca, Antonio Angelo Martins da (2005) Instituiçâo e Desenvolvimento Territorial. $O$ desempenho municipal após a descentralização. Feira de Santana: UEFS.

Fonseca, Antonio Angelo Martins da (2013) "Federalismo, descentralização e localismo no Brasil”, en A. A. M. de Fonseca (ed.) Estado, territórios e a dinâmica das fronteiras: reflexões e novos desafios. Salvador: JM Gráfica e Editora; Salvador: Universidade Federal da Bahia; Guarda/Portugal: Centro de Estudos Ibéricos (SEI); Coimbra/Portugal: Centro de Estudos de Geografia e Ordenamento do Território (CEGOT); Santiago de Compostela: Universidade de Santiago de Compostela, 159-186.

García Delgado, Daniel (1997) Estado y sociedad: la nueva relación a partir del cambio estructural. Buenos Aires: Norma.

Governo da Bahia (2003) Plano Plurianual da Bahia 2004-2007. Salvador de Bahia: Seplantec.

Governo da Bahia (2007) Plano Plurianual da Bahia 2008-2011. Salvador de Bahia: Seplan.

Haersbaert, Rogério (2007) “Concepçoes do território para entender a desterritorializaçao”, en M. Santos y B. Becker (orgs.) Território, territórios, ensaios sobre o ordenamento territorial. Rio de Janeiro: Lamoarina, 43-72.

Haersbaert, Rogério (2011) O mito da desterritorializaçao: do "fim dos terrtorios" á multiterritorialidade. Rio de Janeiro: Bertrand Brasil.

Ibañez, Pablo (2007) Território e guerra fiscal: a perversidade dos incentivos territoriais. Disertación de Mestrado em Geografia. Faculdade de Filosofia Letras e Ciências Humanas da Universidade de São Paulo, São Paulo.

Martin, André Roberto (2005) “Dilemas do federalismo: entre o «estadualismo oligárquico» e o «regionalismo burocrático»”. Perspectivas (São Paulo), núm. 27, 55-68.

Montesquieu, Charles Louis de Secondat, Barón de (2001 [1748]) O espírito das leis. [Puesto en línea el 5 de junio de 2001. URL: <http://www.dhnet.org.br/direitos/anthist/marcos/hdh_montesquieu_o_espirito_das_leis .pdf $>$. Consultado el 6 diciembre de 2014].

Paasi, Anssi (2002) "Place and Regions: regional worlds and words". Progress in Human Geography, vol. 26, núm. 6, 802-811.

Pessoti, Gustavo Casseb; Sampaio, Marcos Guedes Vaz, y Spínola, Noélio Dantaslé (2008) “Incentivos fiscais e a política de atração industrial na Bahia: uma análise pós 1990”. Revista Desenbahia (Salvador), núm. 8, 7-23.

Rafestin, Claude (1993) Por una geografia del poder. Zamora (Michoacán): El Colegio de Michoacán.

Rezende, Fernando (1995) “Federalismo Fiscal no Brasil”. Revista de Economia Política (São Paulo), núm. 15, 1-22.

Rezende, Fernando (2006) Desafios do federalismo fiscal. Rio de Janeiro: FGV.

Rezende, Fernando (2009) A Reforma Tributária e a Federação. Rio de Janeiro: FGV. 
Rezende, Fernando, y Afonso, José Roberto (2004) “A federação brasileira: desafios e perspectivas”, en F. Rezende, F. A. de Oliveira y E. Araújo (eds.) Federalismo e integração econômica regional: desafios para o Mercosul. Rio de Janeiro: Konrad Adenauer Stiftung, 301-361.

Rezende, Fernando; Oliveira, Fabrício Augusto de, y Araujo, Erika (2007) O dilema fiscal: remendar ou reformar? Rio de Janeiro: FGV.

Rios, Ricardo Bahia (2014) "Resgatando a origem do federalismo moderno: o caso brasileiro e seus reflexos na gestão municipal”. Revista Caminhos de Geografia, vol. 15, núm. 52, 162-172.

Rodrigues, Denise Andrade (1998) “O Papel dos Governos Estaduais na Indução do Investimento: A Experiência dos Estados do Ceará, Bahia e Minas Gerais”. Revista do BNDES, núm.10, 1-33.

Sano, Hironobu, y Abrucio, Fernando Luiz (2011) “A experiência de cooperação interestadual no Brasil: formas de atuação e seus desafios”. Cadernos Adenauer (São Paulo), núm. 4, 91-110.

Santos, Milton (2002) Por uma outra globalização: do pensamento único à consciência universal. Rio de Janeiro: Record.

Santos, Milton (2006a) “O dinheiro e o território”, en M. Santos (ed.) Território, territórios: ensaios sobre o ordenamento territorial ( $2^{\mathrm{a}}$ ed.). Rio de Janeiro: DP\&A.

Santos, Milton (2006b) A natureza do espaço: técnica e tempo, razão e emoção. São Paulo: Hucitec.

Silva, Sylvio Bandeira de Mello; Silva, Barbara-Christine Nentwig, y Coelho, Araori Silva (2008) Desequilíbrios e desigualdades no Brasil e nos Estados Brasileiro. João Pessoa: Grafset.

Silva, Sylvio Bandeira de Mello, y Fonseca, Antonio Angelo Martins da (2008) "Territoriais de integração e fortalecimento urbano-regional para o Estado da Bahia”. Revista de Desenvolvimento Econômico (Salvador), vol. X, núm. 17, 12-22.

Soares, Márcia Miranda (1998) “Federação, democracia e instituições políticas”. Lua Nova, núm. 44, 137-163.

Souza, Celina (2003) "Federalismo e Conflitos Distributivos: Disputa dos Estados por Recursos Orçamentários Federais”. DADOS - Revista de Ciências Sociais (Rio de Janeiro), vol. 46, núm. 2, 345-384.

Souza, Celina (2005) "Federalismo, desenho constitucional e instituições federativas no Brasil pós-1988”. Revista de Sociologia e Política (Curitiba), núm. 24, 105-122.

Souza, Celina (2008) "Federalismo: Teorias e Conceitos Revisitados”. Revista Brasileira de Informação Bibliográfica em Ciências Sociais, núm. 65, 27-48.

Souza, Celina (2013) "Para entender a nossa barafunda federativa”. Insight Inteligência (Rio de Janeiro), vol. 61, 60-70.

Taylor, Peter J., y Flint, Colin (2002) Geografía política: Economía Mundo, Estado Nación y Localidad. Madrid: Trama Editorial.

Valdés, Roberto Blanco (2012) Los rostros del federalismo. Madrid: Alianza Editorial.

Vieira, Vanessa da Silva (2009) "Modelo de desenvolvimento regional do estado da Bahia: análise a partir do plano plurianual”, en Anais do VIII Encontro Nacional da Associação Nacional de Pós-Graduação e Pesquisa em Planejamento Urbano e Regional (ENANPUR). Florianópolis: UFSC, 25 pp. 